\title{
Osteoporosis in a Rat Model Co-Exposed to Cigarette Smoke and Intermittent Hypoxia
}

This article was published in the following Dove Press journal:

International Journal of Chronic Obstructive Pulmonary Disease

\author{
Yan Zhuang \\ Yuxia Yan \\ Xia Yang \\ Jie Cao
}

Department of Respiratory and Critical Care Medicine, Tianjin Medical University General Hospital, Tianjin, People's Republic of China
Correspondence: Xia Yang; Jie Cao Department of Respiratory and Critical Care Medicine, Tianjin Medical University General Hospital, Tianjin 300052, People's Republic of China

Tel +86 I3682 I873 I9;

$+8613132088076$

Fax +862260361720

Email xiaoxia20050III@163.com;

tjcaojie@sina.com
Purpose: There are few studies on osteoporosis in chronic obstructive pulmonary diseaseobstructive sleep apnea overlap syndrome, and the results obtained are inconsistent. The purpose of our study is to observe the occurrence of osteoporosis and its possible mechanism in rat model co-exposed to cigarette smoke and intermittent hypoxia.

Materials and Methods: The rats were randomly divided into four groups: air exposed group, cigarette smoke (CS) exposed group, 10\% concentration of intermittent hypoxia exposed group, CS combined with $10 \%$ concentration of intermittent hypoxia exposed group. All animals completed lung function and lung tissue morphology assessment. The femurs were examined by microcomputer tomography (microCT). Tartrate-resistant acidic phosphatase (TRAP) staining was used to evaluate the osteoclasts. We also assessed the interleukin-6 (IL-6) and tumor necrosis factor- $\alpha$ (TNF- $\alpha$ ) in peripheral blood.

Results: There was no difference in the femoral length between each group, but the quantitative analyses of microCT showed that compared with the air exposed group, the percent bone volume (BV/TV), trabecular thickness (Tb.Th), trabecular number ( $\mathrm{Tb}$. $\mathrm{N}$ ), cortical thickness (Ct.Th) and bone mineral density (BMD) decreased, and the trabecular separation (Tb.Sp) and the proportion of trap-positive cells increased significantly in the overlapping exposed group. There were higher levels of BV/TV in the overlapping group than CS exposed group. Compared with the intermittent hypoxia exposed group, there were lower levels of $\mathrm{Tb} . \mathrm{Th}$ and Ct.Th and higher levels of Tb.Sp in the overlapping exposed group. However, there was no statistical difference of trap-positive cell between the overlapping exposed group and the CS exposed single group or the intermittent hypoxia exposed group. There were higher levels of IL-6 and TNF- $\alpha$ in the overlapping exposed group than those in the air-exposed group.

Conclusion: Bone destruction increased in the overlapping exposed rat model compared with the rat exposed to air, which may be related to the upregulation of inflammation.

Keywords: osteoporosis, cigarette smoke exposure, intermittent hypoxia exposure, overlapping exposed rat model

\section{Introduction}

Chronic obstructive pulmonary disease (COPD) and obstructive sleep apnea (OSA) are common respiratory diseases, and the coexistence of both diseases is called overlap syndrome. Overlap syndrome patients have more severe nocturnal oxygen desaturation and worse prognosis. ${ }^{1-3}$ These patients may have a higher risk of comorbidities than patients with COPD or OSA alone, such as cardiovascular diseases, pulmonary hypertension. ${ }^{4-6}$ Oxidative stress and systemic inflammation are the common pathological mechanisms of COPD or OSA which may play an essential role in the occurrence of complications in overlap syndrome patients. ${ }^{4,6-9}$ 
Osteoporosis is a common chronic systemic disease with reduced bone mass, destruction of bone microstructure and fragility. Common causes of osteoporosis include age, gender, diet and steroid application. ${ }^{10}$ Previous studies have found that the incidence of osteoporosis in COPD was high, about $9-69 \%{ }^{11}$ OSA also has adverse effects on bone, and studies revealed that OSA was a factor leading to osteoporosis. ${ }^{12}$ The studies also found that OSA may be the risk factor of osteoporosis in COPD. ${ }^{13}$

However, there are few studies on osteoporosis in overlap syndrome and the results obtained are not yet established. Bone disease is rarely taken into consideration during the COPD-OSA overlap syndrome patients. There is a lack of research on osteoporosis in cigarette smoke exposed or intermittent hypoxia exposed animal models, especially the lack of research in overlapping exposed models. The main purpose of our study is to observe the occurrence of osteoporosis and its possible mechanism in the overlapping exposed rat model.

\section{Materials and Methods}

\section{Animals}

Male wild-type Wistar rats (6 weeks of age, 120-140g body weight) were purchased from Beijing Vital River Laboratory Animal Technology Co., Ltd. (Beijing, China), and raised in the clean and sterile second level animal room. The animal study was conducted following the specifications of Animal Ethical and Welfare of Tianjin Medical University, and was approved by the Ethical Committee of Tianjin Medical University General Hospital (IRB2020-KY-232).

\section{Cigarette Smoke Exposure and Intermittent Hypoxia Exposure}

All the rats were divided randomly into four groups containing 6 rats at each group: air exposed group (Air), cigarette smoke exposed group (CS), 10\% concentration of intermittent hypoxia exposed group (IH10\%), CS combined with $10 \%$ concentration of intermittent hypoxia exposed group (CS+IH10\%).

The rats in the Air group were regularly raised in the air. The rats in the CS group were exposed to active smoke produced by commercial cigarettes (Daqianmen cigarettes with filter purchased from Shanghai, China; tar $10 \mathrm{mg}$, nicotine $1 \mathrm{mg}, \mathrm{CO} 12 \mathrm{mg}$ ) in homemade smoke exposure device as described previously. ${ }^{14}$ By burning 15 cigarettes at the same time, the volume fraction of smoke in the rearing cabin was about 15\% (V/V). Animals were exposed 1 hour twice a day at 7:30-8:30 and 17:30-18:30, 6 days a week, a total of 16 weeks.

The rats in the $\mathrm{IH} 10 \%$ group were placed in the homemade IH cabin from 9:00 to 17:00 (sleep period), 6 days a week, a total of 16 weeks. Intermittently filled the cabin with pure nitrogen for $30 \mathrm{~s}$ ( $6 \mathrm{~L} / \mathrm{min}$ per minute). The oxygen concentration in the cabin gradually reduced to a minimum of $10 \%$, and then charged compressed air for $90 \mathrm{~s}(15 \mathrm{~L} / \mathrm{min}$ per minute) to make the oxygen concentration of cabin gradually increased to a maximum of $20.9 \%$ and maintained for a certain period, 2 minutes were a cycle. The above was decomposed by a gas control delivery system as described previously. ${ }^{14}$ The rats in CS $+\mathrm{IH} 10 \%$ group were exposed to intermittent hypoxia between two cigarette smoke exposures on the same day.

\section{Lung Function Tests}

After 16 weeks of exposure, all animals completed lung function tests. Rats were fully anaesthetized with $10 \%$ chloral hydrate $(0.3 \mathrm{~mL} / 100 \mathrm{~g})$ intraperitoneal injection. After tracheotomy and intubation, the tracheal cannula was connected to a computer-controlled whole-body plethysmograph system (AniRes 2005 Lung Function System Version 3.5, Bestlab, Beijing, China). Completed the operation according to the user manual, got and recorded the results, including forced expiratory volume (FEV), expiratory resistance (Re).

\section{Lung Histomorphological Assessment}

The left lung tissues of the rats were taken and fixed with $4 \%$ paraformaldehyde, then embedded in paraffin, sectioned and stained with hematoxylin and eosin staining (HE). Image J (NIH Image, Bethesda, MD, USA) was used for image analysis. Using the mean linear intercept $(\mathrm{Lm})$ and destructive index (DI) to evaluate the rat airspace enlargement and alveolar wall destruction, respectively. ${ }^{15,16}$

\section{Microcomputed Tomography of Femurs}

The femurs were obtained and fixed with $4 \%$ paraformaldehyde. A professional technician used the microcomputer tomography (microCT) (SkyScan 1276, Kontich, Belgium) to scan the femurs. The scan condition including an X-ray tube potential of $85 \mathrm{kV}$, an X-ray intensity of $200 \mu \mathrm{A}$, an exposure time of $384 \mathrm{~ms}$ and an isotropic voxel size of $6.53 \mu \mathrm{m}$. Used Nrecon software (version 1.7.4.2) for threedimensional reconstruction and CT-Analyzer software (version 1.18.8.0) for data analysis. Analyses of the bone micro architecture were performed in a region of interest (ROI), which was set to a region having the same height of $0.5 \mathrm{~mm}$ 
and a length of $2 \mathrm{~mm}$ from the proximal border of the growth plate at the same gray value. Got the following results of software analysis: percent bone volume (BV/TV), trabecular thickness (Tb.Th), trabecular number (Tb.N), trabecular separation (Tb.Sp), cortical bone thickness ( $\mathrm{Ct} . \mathrm{Th})$, bone mineral density (BMD).

\section{Tartrate-Resistant Acidic Phosphatase Staining of Femoral Tissues}

The 4\% paraformaldehyde fixed femurs decalcified in $10 \%$ EDTA, then embedded in paraffin and sectioned. The osteoclasts were detected using the kit of tartrateresistant acidic phosphatase (TRAP) staining (Servicebio, Wuhan, China) according to the manufacturer's protocol. The osteoclasts were stained red, and the nuclei were light blue. The results were quantified by counting the number of positive cells per bone perimeter (B.Pm) using the Image $\mathrm{J}$ (NIH Image, Bethesda, MD, USA).

\section{Cytokine Detection}

The serum levels of interleukin -6 (IL-6) and tumor necrosis factor- $\alpha(\mathrm{TNF}-\alpha)$ were measured with enzyme-linked immunosorbent assay (ELISA) kits (Abcam, Cambridge, MA, USA) according to the manufacturer's instructions.

\section{Statistical Analysis}

All data were analyzed using the SPSS21.0 software (Statistics Package for the Social Sciences, Inc., Chicago, IL, USA). Data were presented as mean $\pm \mathrm{SD}$. Mann-Whitney $U$-test was used for comparison between groups. $P$-value $<0.05$ was defined as the statistically significant difference.

\section{Results}

\section{Evaluation of Lung Function and Lung Tissue Morphology in Each Group}

Following the 16 weeks of exposure, all animals completed lung function and lung morphometric assessment. Compared with the group exposed to air only, Re of lung function in the CS exposure group increased, and FEV decreased significantly (Figure 1A and B). At the same time, lung tissue sections after HE staining showed that the Lm and DI in the CS exposed group were significantly increased (Figure 1C-F). We can see the increased inflammatory cell infiltration around the airways in the CS exposed group (Figure 1E). The above all indicated the successful induction of COPD model.

In the CS combined intermittent hypoxia (10\%) exposed group, we also found a significant decrease in lung function, and the considerable increase in Lm and DI of lung tissue compared to the air exposed group (Figure 1A-D). We also observe significant inflammatory cell infiltration around the airways and alveolar structure destruction in the overlapping exposed group (Figure $1 \mathrm{E}$ and $\mathrm{F}$ ). However, there were no significant differences in all parameters between the overlapping exposed group and the CS exposed group.

Compared with the air exposed group, only the Lm increased significantly in the intermittent hypoxia exposed group, and the differences of others were not statistically significant (Figure 1A-D). We also compared the difference between the intermittent hypoxia alone exposed group and the overlapping exposed group, only found an increase in DI in the overlapping group (Figure 1D).

\section{Changes of Bone Microarchitecture} Revealed by microCT

To understand the changes of bone microarchitecture in the overlapping exposed rat model, microCT of femurs were used for evaluation. Three-dimensional reconstruction of cortical bones and trabecular bones showed that the bone microstructure of the overlapping exposed group changed significantly compared with the air exposed group, becoming thinner and fewer trabeculae (Figure 2A).

We compared the femoral length between each group, found no noticeable difference (Figure 2B). The quantitative analyses of microCT showed that compared with the air exposed group, the BV/TV, Tb.Th, Tb.N, Ct.Th, and BMD decreased and Tb.Sp increased significantly in either overlapping exposed group or cs exposed group (Figure 2C-H). The bone structure changed significantly in both cs exposed group and overlapping group. We also found that compared with the air exposed group, the intermittent hypoxia exposed group had lower levels of BV/TV and Tb.N, but there was no significant difference in Tb.Th, Tb.Sp, Ct.Th and BMD between the two groups (Figure $2 \mathrm{C}-\mathrm{H}$ ).

There were higher levels of BV/TV in the overlapping group than cs exposed group. We also found that compared with the intermittent hypoxia exposed group, the levels of Tb.Th, Ct.Th decreased and Tb.Sp increased significantly in the overlapping exposed group (Figure 2C-H).

\section{Osteoclasts Quantitative Analyses in Bone Tissue}

Osteoclasts are important indicators for evaluating bone degradation, and we performed TRAP staining on paraffinembedded sections of the femur for the osteoclastic 
A
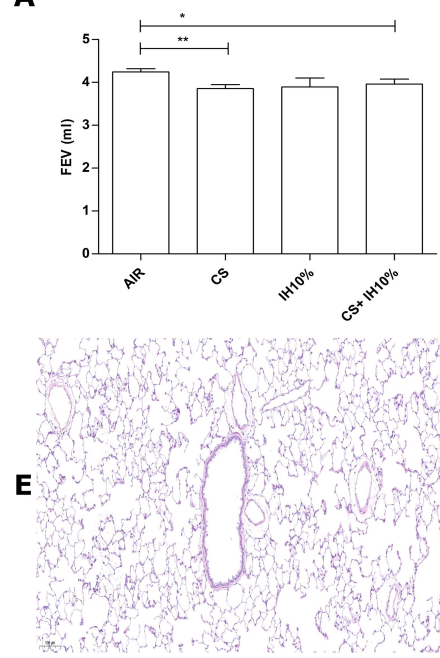

AIR

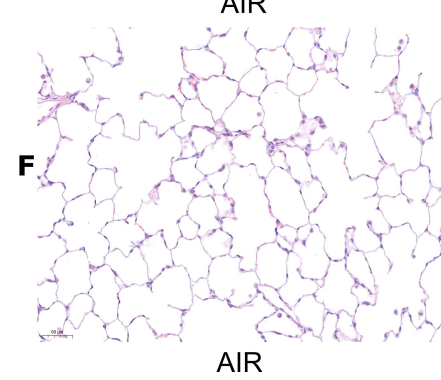

B
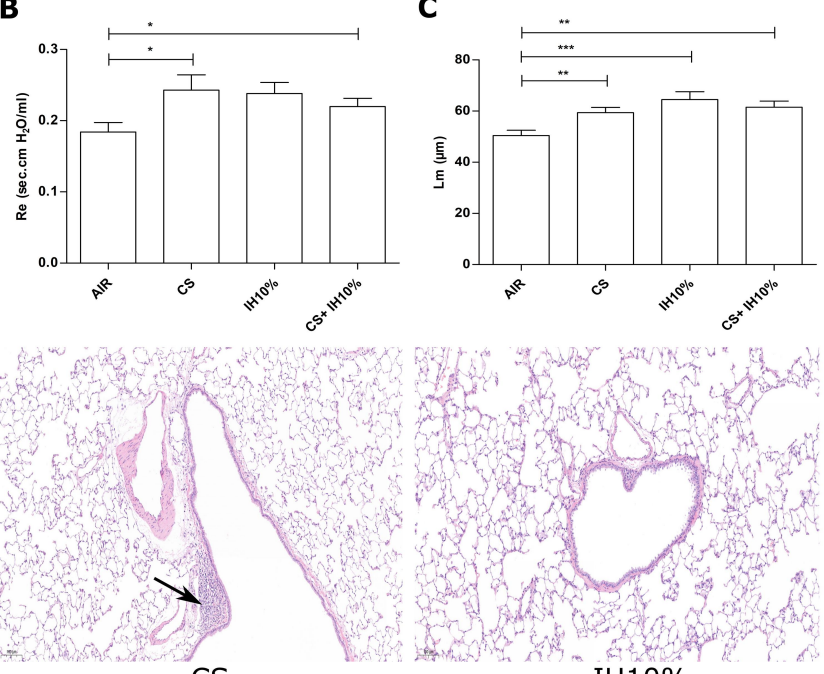

$\mathrm{IH} 10 \%$

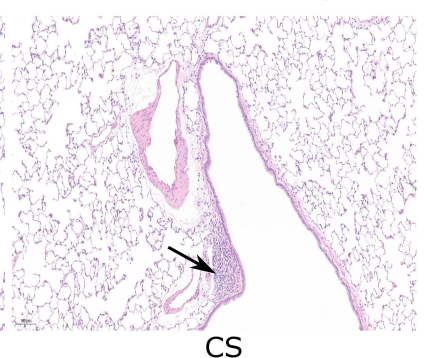

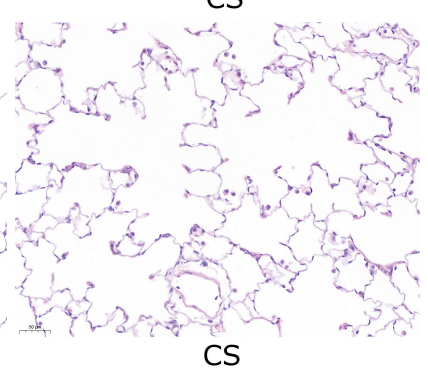

CS

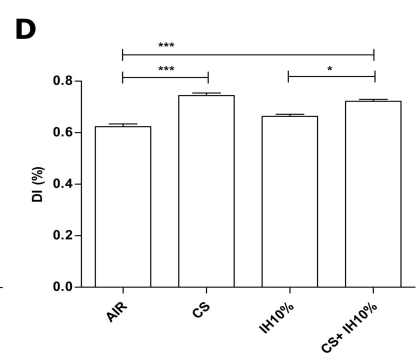

D

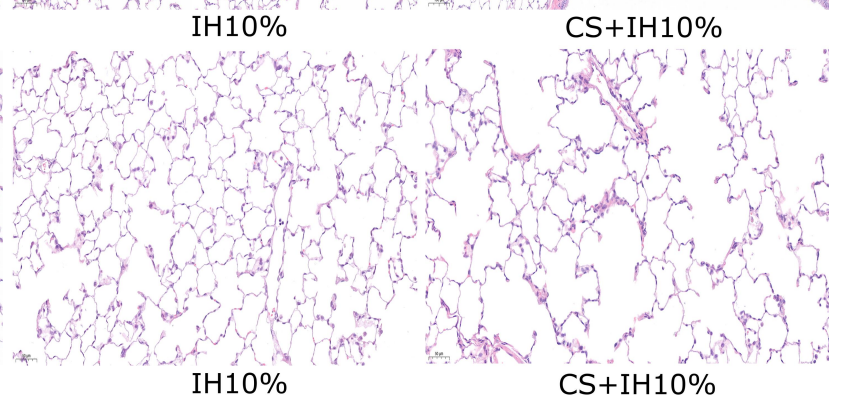

Figure I Lung function and Lung histomorphological. (A) Forced expiratory volume (FEV) and (B) expiratory resistance (Re) of each group. (C) Mean linear intercept (Lm) $($ Scale bar $=100 \mu \mathrm{m})$ and $(\mathbf{D})$ destructive index (DI) (Scale bar $=50 \mu \mathrm{m})$ of each group. (E-F) Hematoxylin and eosin staining of rat lung tissue in each group. Arrows indicate inflammatory cell infiltration. Data presented as mean \pm SD ( $n=6$ per group). * $P<0.05$; ** $P<0.01$; *** $P<0.001$.

activity in different groups (Figure 3A). Compared with the air exposed group, the proportion of positive cells increased significantly in the smoke exposed group, the intermittent hypoxia exposed group, and the overlapping exposed group. However, there was no statistical difference between the overlapping exposed group and the CS exposed single group or the overlapping exposed group and the intermittent hypoxia exposed group (Figure 3B). These data suggest that smoke exposure and intermittent hypoxia exposure are both related to osteoclastogenesis, but there is no enhancement after overlapping exposure.

\section{The Expression of Inflammatory Cytokines in Peripheral Blood of Each Group}

Upregulation of inflammatory cytokines are associated with osteoporosis. Therefore, we also detected the serum levels of IL- 6 and TNF- $\alpha$ (Figure 4A-B). We found that the levels of IL- 6 and TNF- $\alpha$ in the overlapping exposure group were both significantly higher than those in the air exposed group. At the same time, the levels of IL-6 and
TNF- $\alpha$ in the overlapping group increased significantly than that in the intermittent hypoxia exposed group. There was no significant difference between the CS exposed group and the overlapping exposed group.

\section{Discussion}

Previous clinical studies have found that COPD patients with OSA have lower bone mineral density than patients with COPD alone. It may be associated with the increase of systemic inflammation and the decrease of exercise ability in COPD-OSA overlap syndrome patients. Oxygen desaturation index is an independent factor related to bone mineral density in patients with COPD. ${ }^{13}$ But our study is the first to observe osteoporosis in cigarette smoke and intermittent hypoxia overlapping exposed animal models. We found that compared with air exposed group, bone loss was more evident in overlapping animal models, and that may be correlated with the up-regulation of systemic inflammation. Besides, our results also found that bone loss may be reduced in the overlapping exposed group compared with the CS exposed group and bone loss may increase compared with the intermittent hypoxia 


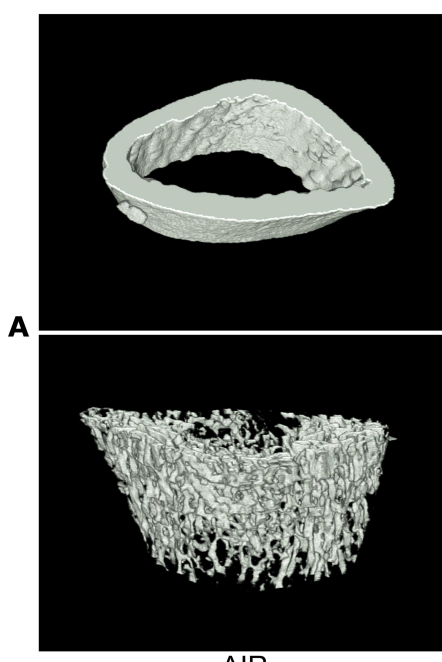

AIR

B

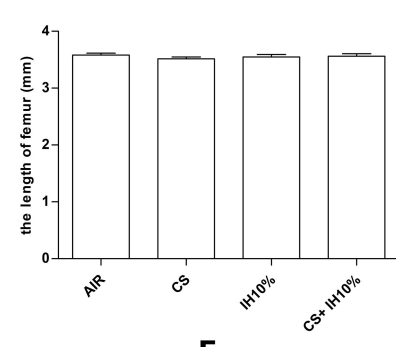

F

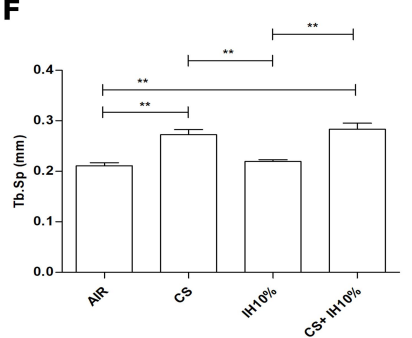

C
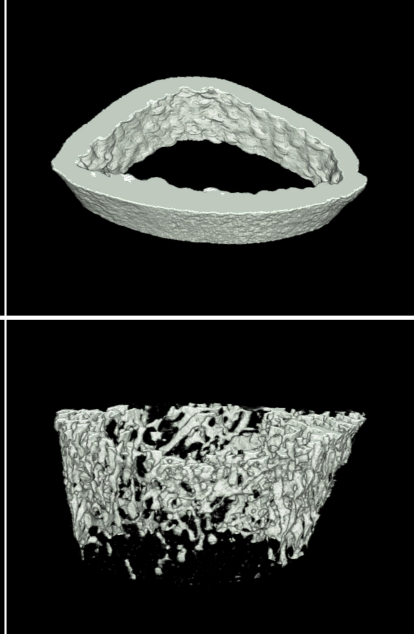

CS

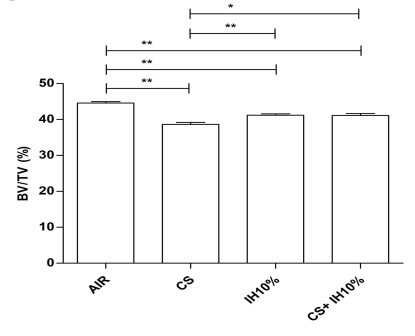

D

G

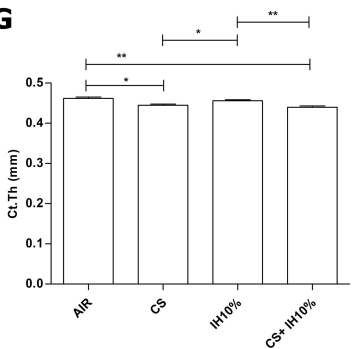

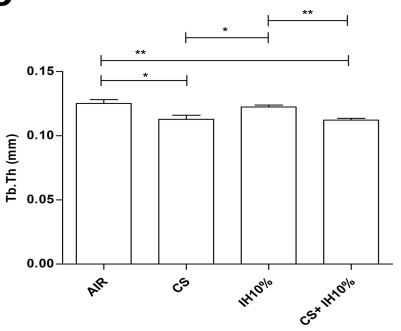
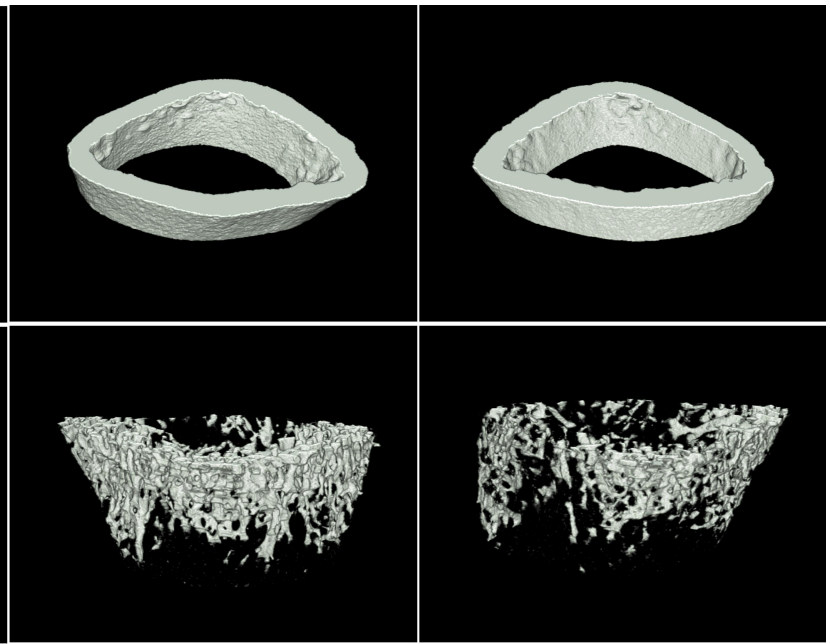

IH10\%

$\mathbf{E}$

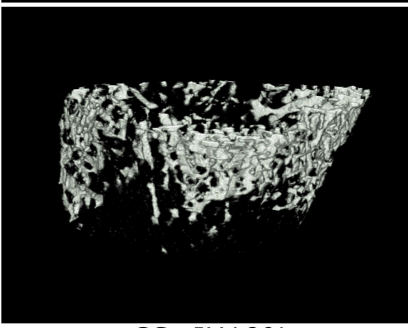

$\mathrm{CS}+\mathrm{IH} 10 \%$

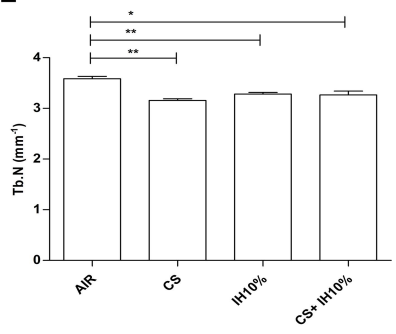

H

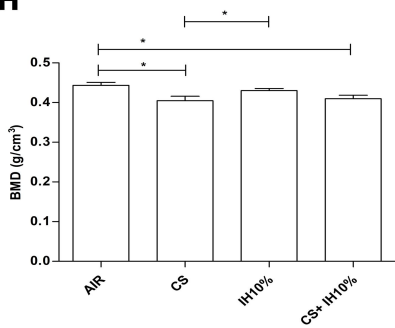

Figure 2 Changes of bone microarchitecture revealed by microcomputed tomography. (A) Three-dimensional reconstruction of cortical bones (upper) and trabecular bones (lower) of the femur. (B) The length of femoral in each group. Quantitative analyses of parameters including: (C) percent bone volume (BV/TV), (D) trabecular thickness (Tb.Th), (E) trabecular number (Tb.N), (F) trabecular separation (Tb.Sp), (G) cortical bone thickness (Ct.Th), (H) bone mineral density (BMD). Data presented as mean \pm SD ( $=5$ per group). $* P<0.05 ; * *<<0.01$.

exposed alone group based on some parameters of bone microarchitecture revealed by microCT. However, there was no statistical difference in the number of trappositive cell between the overlapping exposed group and the CS exposed single group or the overlapping exposed group and the intermittent hypoxia exposed alone group.

In our model, the rats with overlapping exposure had decreased lung function, increased alveolar destruction and infiltration of inflammatory cells around the airway compared with the air exposed group. Although we did not have the blood gas test and animal electroencephalogram monitoring, we used the same modelling method as our research group did before which had been confirmed successfully. ${ }^{14}$ Therefore, the above confirms the successful establishment of rat model co-exposed to cigarette smoke and intermittent hypoxia. In our study, we not only compared the difference between the air exposed group and the overlapping exposed group but also examined the difference between the CS exposed group and the overlapping exposed group or the intermittent hypoxia exposed group and the overlapping exposed group.

Osteoporosis is a systemic skeletal disease and usually related to age, sex, low weight, endocrine disorders and the use of steroid hormones in the general population. ${ }^{10}$ The incidence of osteoporosis in COPD patients is significantly higher than that in the general population, and it is related to worse health status and prognosis. ${ }^{11,17,18}$ Osteoporosis is one of the common comorbidities of COPD. ${ }^{19}$ Animal models 

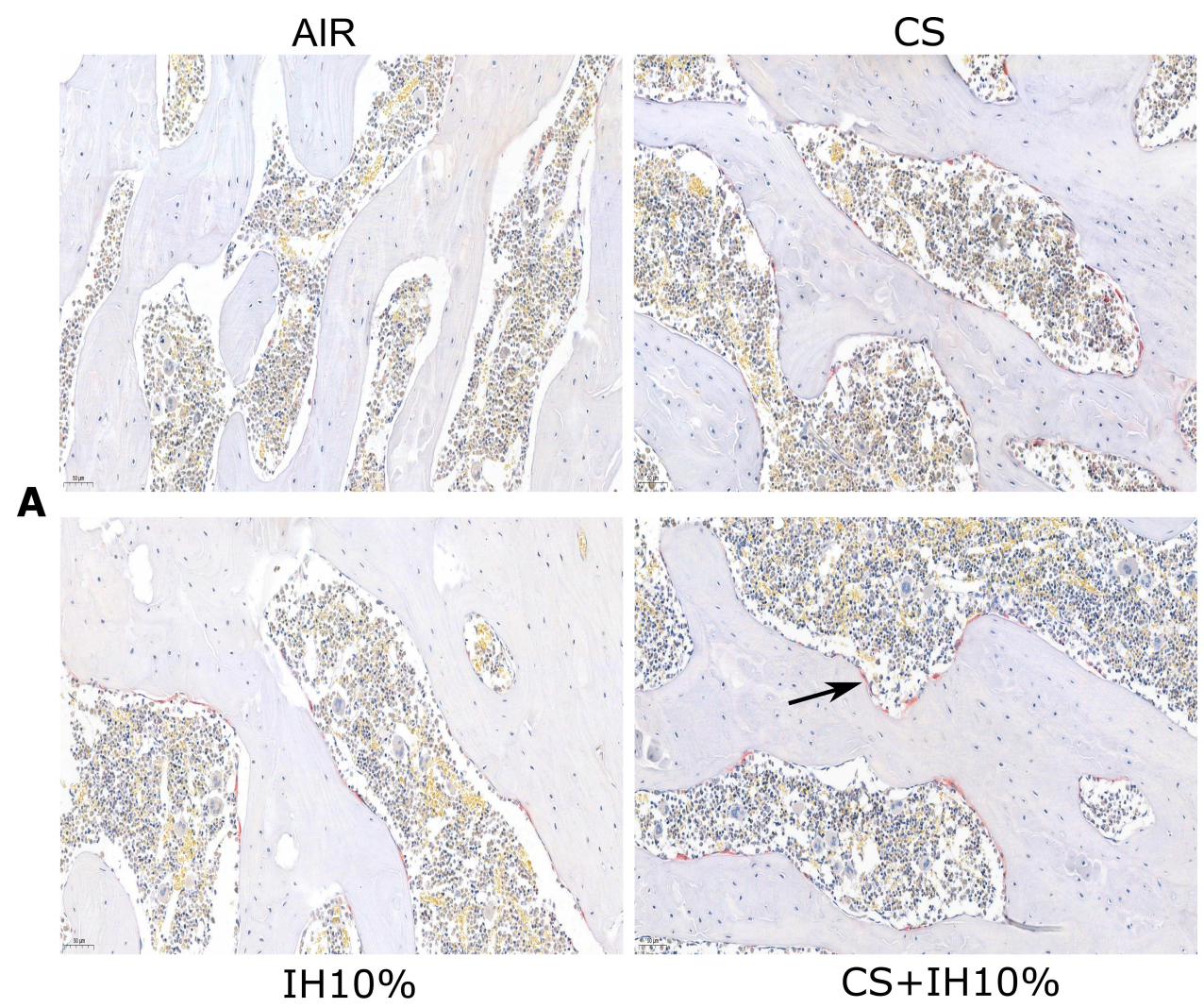

IH $10 \%$

$\mathrm{CS}+\mathrm{IH} 10 \%$

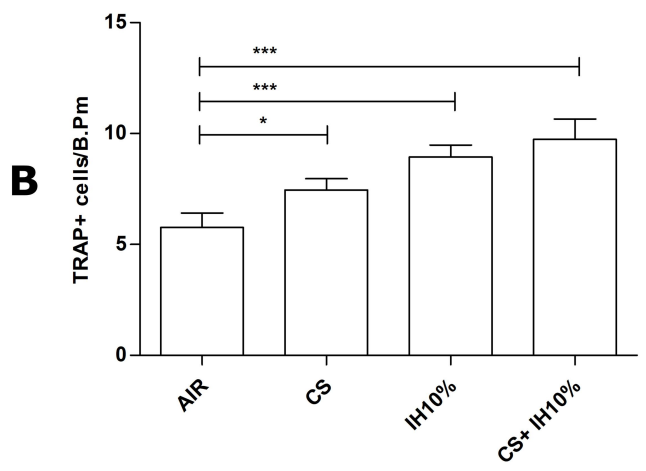

Figure 3 TRAP staining and quantitative analyses of osteoclasts in femoral tissues. (A) TRAP staining of femoral tissues in each group (Scale bar=50 $\mu \mathrm{m})$. TRAP-positive osteoclasts were stained red, and the nuclei were light blue. Arrows indicate TRAP-positive osteoclasts. (B) Numbers of TRAP-positive osteoclasts, measured as cells per millimeter of perimeter (cells/B.Pm). Data presented as mean \pm SD ( $n=5$ per group). $* P<0.05 ; * * * P<0.00$ I.

have also confirmed that chronic smoke exposure is associated with osteoporosis. $^{20-22}$ In our study, we found that the bone microarchitecture was degraded and the proportion of osteoclasts increased obviously in CS exposed group than that in air exposed group. These are consistent with the previous results.

OSA is associated with many diseases, including osteoporosis. Due to the different study designs, the current relationship between OSA and osteoporosis is not entirely consistent. $^{23}$ The previous cohort studies had found that OSA was associated with increased bone resorption and decreased BMD. ${ }^{12,24-26}$ However, there was also a study found that the risk of osteoporosis in elderly patients with OSA didn't increase, and even intermittent hypoxia may reduce the occurrence of osteopenia/osteoporosis in the elderly. ${ }^{27}$ At the same time, there were few studies of osteoporosis in chronic intermittent hypoxia animal models. In our animal model study, we found that compared with the air exposed group, the $10 \%$ intermittent hypoxia exposed group had lower levels of BV/TV and Tb.N, but there was no significant difference of Tb.Th, Ct.Th, Tb.Sp and BMD between the two groups. Our findings are consistent with the previous research by Marta Torres. They 
A

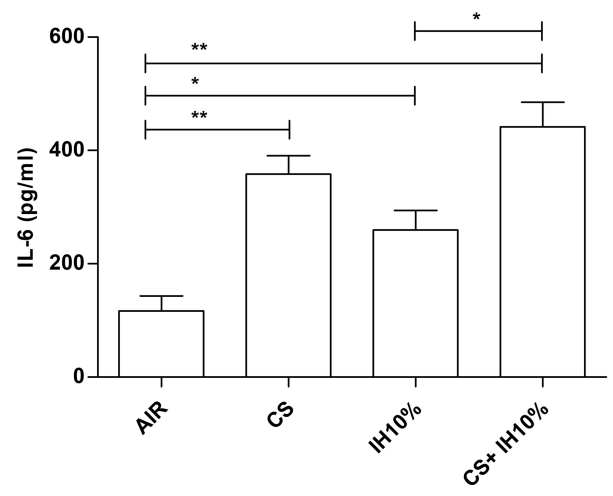

B

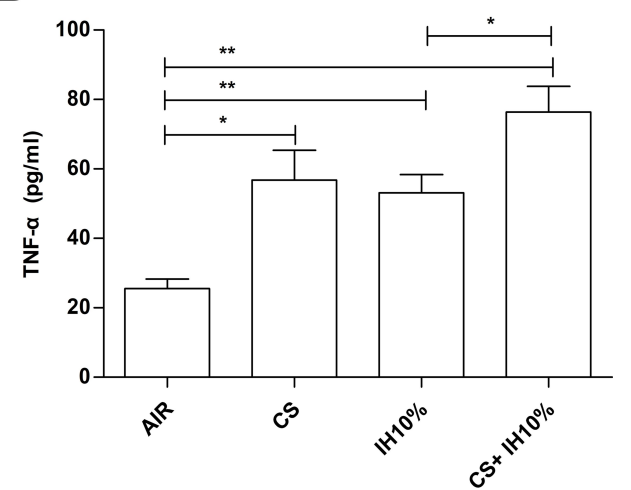

Figure 4 Concentration of IL-6 and TNF- $\alpha$ in peripheral blood of each group. Levels of serum (A) IL-6 and (B) TNF- $\alpha$ were measured with ELISA. Data presented as mean $\pm \mathrm{SD}$ ( $\mathrm{n}=5$ per group). $* P<0.05 ; * *<<0.01$.

found that after 32 days of 5\% intermittent hypoxia exposure, intermittent hypoxia did not significantly change BMD. ${ }^{28}$ In our study, we found that the proportion of osteoclasts in bone tissue increased significantly in the intermittent hypoxia exposed group than the control group. The above results suggest that the bone loss in the intermittent hypoxia exposed group might be more obvious than that in the air exposed group. More animal experiments are still needed to verify it.

In our study, we found that the changes in bone microarchitecture in the overlapping exposed group were more apparent than that in the air exposed group, the proportion of osteoclasts were significantly increased. The results suggest that overlapping exposure leads to increased bone loss. The previous study found that hypoxia itself can directly promote bone resorption by increasing the formation of osteoclasts. $^{29}$ At the same time, it has been established that smoking can also cause osteoporosis. ${ }^{30}$ Our research found that the proportion of trap positive cells was significantly increased in the smoke exposed group and the intermittent hypoxia exposed group compared with the air exposure group. We found that there were higher levels of BV/TV in the overlapping group than cs exposed group, but the levels of Tb.Th, Ct.Th decreased and the levels of Tb.Sp increased significantly in the overlapping group compared with intermittent hypoxia exposed group. And there was no statistical difference in the number of trap-positive cell between the overlapping exposed group and the other two groups exposed alone. Based on the above results, we can't draw the conclusion that there is significant change in bone loss in the overlapping group compared to the individual exposed groups, which needs to be confirmed by more studies.
To discuss the possible mechanism of osteoporosis in the overlapping rat model, we compared the levels of blood inflammatory cytokines. We found that the levels of IL-6 and TNF- $\alpha$ in the overlapping exposed group were significantly higher than those in the air exposed group. Both IL-6 and TNF- $\alpha$ are involved in promoting osteoclast formation. $^{31-35}$ Therefore, we speculate that the upregulated inflammatory cytokines in the overlapping group may play an important role in the formation of osteoporosis in overlapping exposed rat model. Previous studies have shown that the link between proinflammatory factor and osteoclastogenic cytokine release plays an important role in osteoporosis. ${ }^{36}$ Interestingly, osteoporosis is one of the systemic complications associated with COPD and COPD-OSA overlapping syndrome, respiratory diseases with significantly increased levels of pro-inflammatory factor. ${ }^{37}$ Both of these studies support our hypothesis that the osteoporosis formation was a result of inflammatory and osteoclastogenic cytokine secretion in the rat model co-exposed to cigarette and intermitted hypoxia. But in our study, we also found that compared with the intermittent hypoxia exposed group, the levels of IL-6 and TNF- $\alpha$ in the overlapping group were higher and there was also decreased Tb.Th, Ct.Th and increased $\mathrm{Tb}$.Sp, but there was no difference in the number of trap-positive cells between the two groups. It suggests that there may be other mechanisms involved in the occurrence of osteoporosis in overlapping exposed rats.

Our study has some limitations: 1) Our research was only an observational study and didn't thoroughly explore the mechanism of osteoporosis in the overlapping model. Because smoking itself can cause osteoporosis, it is not clear whether osteoporosis in the overlapping model is 
related to pathological changes of the lung. 2) Although rat model co-exposed to cigarette smoke and intermittent hypoxia was successfully established in our study, it is unclear whether the occurrence of osteoporosis in the overlapping model is related to the smoke exposure time, the intermittent hypoxia exposure time, the concentration of intermittent hypoxia, the age or the gender of rats, that will be our further research direction.

\section{Conclusion}

Bone destruction in the rat model of overlapping exposure increased significantly, which may be related to the coexposure of cigarette smoke and intermittent hypoxia, and the upregulation of inflammatory cytokines. Osteoporosis may be one of the complications of COPD-OSA overlap syndrome.

\section{Acknowledgments}

The authors acknowledge the economic support from the Tianjin Natural Science Foundation of China (18JCQNJC82900), the National Natural Science Foundation of China (81670084, 81600067, 81970084), and the 13th Five-Year Plan for First-Class Discipline Construction of China.

\section{Author Contributions}

All authors contributed to data analysis, drafting or revising the article, have agreed on the journal to which the article will be submitted, gave final approval of the version to be published, and agree to be accountable for all aspects of the work.

\section{Disclosure}

The authors report no conflicts of interest in this work.

\section{References}

1. Md Shawon SR, Perret JL, Senaratna CV, Lodge C, Hamilton GS, Dharmage SC. Current evidence on prevalence and clinical outcomes of co-morbid obstructive sleep apnea and chronic obstructive pulmonary disease: a systematic review. Sleep Med Rev. 2017;32:58-68.

2. Sanders MH, Newman AB, Haggerty CL, et al. Sleep Heart Health Study. Sleep and sleep-disordered breathing in adults with predominantly mild obstructive airway disease. Am J Respir Crit Care Med. 2003;167(1):7-14. doi:10.1164/rccm.2203046

3. Marin JM, Soriano JB, Carrizo SJ, Boldova A, Celli BR. Outcomes in patients with chronic obstructive pulmonary disease and obstructive sleep apnea: the overlap syndrome. Am J Respir Crit Care Med. 2010;182(3):325-331.

4. McNicholas WT. COPD-OSA Overlap Syndrome: evolving evidence regarding epidemiology, clinical consequences, and management. Chest. 2017;152(6):1318-1326. doi:10.1016/j.chest.2017.04.160
5. Owens RL, Malhotra A. Sleep-disordered breathing and COPD: the overlap syndrome. Respir Care. 2010;55(10):1333-1346.

6. McNicholas WT. Chronic obstructive pulmonary disease and obstructive sleep apnea: overlaps in pathophysiology, systemic inflammation, and cardiovascular disease. Am J Respir Crit Care Med. 2009;180(8):692-700. doi:10.1164/rccm.200903-0347PP

7. Agustí AGN, Noguera A, Sauleda J, Sala E, Pons J, Busquets X. Systemic effects of chronic obstructive pulmonary disease. Eur Respir J. 2003;21(2):347-360. doi:10.1183/09031936.03.00405703

8. MacNee W. Oxidants/antioxidants and COPD. Chest. 2000;117 (5Suppl 1):303S-317S. doi:10.1378/chest.117.5_suppl_1.303S-a

9. Ryan S, Taylor CT, McNicholas WT. Selective activation of inflammatory pathways by intermittent hypoxia in obstructive sleep apnea syndrome. Circulation. 2005;112(17):2660-2667. doi:10.1161/ CIRCULATIONAHA.105.556746

10. Prevention and Management of Osteoporosis: report of a WHO scientific group. Available from: https://apps.who.int/iris/handle/ 10665/42841. Accessed 2003.

11. Graat-Verboom L, Wouters EFM, Smeenk FWJM, van den Borne BEEM, Lunde R, Spruit MA. Current status of research on osteoporosis in COPD: a systematic review. Eur Respir J. 2009;34 (1):209-218. doi:10.1183/09031936.50130408

12. Chen Y-L, Weng S-F, Shen Y-C, et al. Obstructive sleep apnea and risk of osteoporosis: a population-based cohort study in Taiwan. J Clin Endocrinol Metab. 2014;99(7):2441-2447. doi:10.1210/ jc.2014-1718

13. Wang T-Y, Yu-Lun L, Chou P-C, et al. Associated bone mineral density and obstructive sleep apnea in Chronic Obstructive Pulmonary Disease. Int $J$ Chron Obstruct Pulmon Dis. 2015;10:231-237. doi:10.2147/COPD.S72099

14. Feng J, Chiang AA-P, Wu Qet al. Sleep-related hypoxemia aggravates systematic inflammation in emphysematous rats. Chin Med $J$ (Engl). 2010;123(17):2392-2399.

15. Saetta M, Shiner RJ, Angus GE, et al. Destructive index: a measurement of lung parenchymal destruction in smokers. Am Rev Respir Dis. 1985;131(5):764-769.

16. Thurlbeck WM. Measurement of pulmonary emphysema. Am Rev Respir Dis. 1967;95(5):752-764.

17. Sabit R, Bolton CE, Edwards PH, et al. Arterial stiffness and osteoporosis in chronic obstructive pulmonary disease. Am J Respir Crit Care Med. 2007;175(12):1259-1265. doi:10.1164/rccm.200701067OC

18. Bolton CE, Ionescu AA, Shiels KM, et al. Associated loss of fat-free mass and bone mineral density in chronic obstructive pulmonary disease. Am J Respir Crit Care Med. 2004;170(12):1286-1293. doi:10.1164/rccm.200406-754OC

19. Global strategy for the diagnosis, management, and prevention of COPD, Global Initiative for Chronic Obstructive Lung Disease (GOLD); 2020. Available from: http://www.goldcopd.org. Accessed November 5, 2019.

20. Sasaki M, Chubachi S, Kameyama N, et al. Effects of long-term cigarette smoke exposure on bone metabolism, structure, and quality in a mouse model of emphysema. PLoS One. 2018;13(1):e191611. doi:10.1371/journal.pone. 0191611

21. Ko CH, Chan RLY, Siu WS, et al. Deteriorating effect on bone metabolism and microstructure by passive cigarette smoking through dual actions on osteoblast and osteoclast. Calcif Tissue Int. 2015;96 (5):389-400. doi:10.1007/s00223-015-9966-8

22. Xiong J, Tian J, Lu Zhou, Yanqing Le, Yongchang Sun. Interleukin17A deficiency attenuated emphysema and bone loss in mice exposed to cigarette smoke. Int $J$ Chron Obstruct Pulmon Dis. 2020;15:301-310. doi:10.2147/COPD.S235384

23. Upala S, Sanguankeo A, Congrete S. Association between obstructive sleep apnea and osteoporosis: a systematic review and meta-analysis. Int $J$ Endocrinol Metab. 2016;14(3):e36317. doi:10.5812/ijem.36317 
24. Tomiyama H, Okazaki R, Inoue D, et al. Link between obstructive sleep apnea and increased bone resorption in men. Osteoporos Int. 2008;19(8):1185-1192. doi:10.1007/s00198-007-0556-0

25. Uzkeser H, Yildirim K, Aktan B, et al. Bone mineral density in patients with obstructive sleep apnea syndrome. Sleep Breath. 2013;17(1):339-342. doi:10.1007/s11325-012-0698-y

26. Hamada S, Ikezoe K, Hirai T, et al. Evaluation of bone mineral density by computed tomography in patients with obstructive sleep apnea. J Clin Sleep Med. 2016;12(1):25-34. doi:10.5664/jcsm.5386

27. Sforza E, Thomas T, Barthélémy J-C, Collet P, Roche F. Obstructive sleep apnea is associated with preserved bone mineral density in healthy elderly subjects. Sleep. 2013;36(10):1509-1515. doi: $10.5665 /$ sleep. 3046

28. Torres M, Montserrat JM, Pavía J, et al. Chronic intermittent hypoxia preserves bone density in a mouse model of sleep apnea. Respir Physiol Neurobiol. 2013;189(3):646-648. doi:10.1016/j.resp.2013. 08.016

29. Utting JC, Robins SP, Brandao-Burch A, Orriss IR, Behar J, Arnett TR. Hypoxia inhibits the growth, differentiation and bone-forming capacity of rat osteoblasts. Exp Cell Res. 2006;312 (10):1693-1702. doi:10.1016/j.yexcr.2006.02.007

30. Iqbal J, Sun L, Cao J, et al. Smoke carcinogens cause bone loss through the aryl hydrocarbon receptor and induction of Cyp1 enzymes. Proc Natl Acad Sci U S A. 2013;110(27):11115-11120. doi:10.1073/pnas. 1220919110
31. Stanojkovic I, Kotur-Stevuljevic J, Spasic S, et al. Relationship between bone resorption, oxidative stress and inflammation in severe COPD exacerbation. Clin Biochem. 2013;46(16-17):1678-1682. doi:10.1016/j.clinbiochem.2013.08.003

32. Raisz LG. Local and systemic factors in the pathogenesis of osteoporosis. World Rev Nutr Diet. 1993;72:92-101.

33. Bertolini DR, Nedwin GE, Bringman TS, Smith DD, Mundy GR. Stimulation of bone resorption and inhibition of bone formation in vitro by human tumour necrosis factors. Nature. 1986;319 (6053):516-518. doi:10.1038/319516a0

34. Boyle WJ, Scott Simonet W, Lacey DL. Osteoclast differentiation and activation. Nature. 2003;423(6937):337-342. doi:10.1038/ nature 01658

35. Manolagas SC, Jilka RL. Bone marrow, cytokines, and bone remodeling. Emerging insights into the pathophysiology of osteoporosis. $N$ Engl J Med. 1995;332(5):305-311. doi:10.1056/NEJM19 9502023320506

36. De Martinis M, Ginaldi L, Sirufo MM, et al. Alarmins in osteoporosis, RAGE, IL-1, and IL-33 pathways: a literature review. Medicina (Kaunas). 2020;56(3):138. doi:10.3390/medicina56030138

37. Gabryelska A, Kuna P, Antczak A, Białasiewicz P, Panek M. IL-33 mediated inflammation in chronic respiratory diseases-understanding the role of the member of IL-1 superfamily. Front Immunol. 2019;10:692. doi:10.3389/fimmu.2019.00692

\section{Publish your work in this journal}

The International Journal of COPD is an international, peer-reviewed journal of therapeutics and pharmacology focusing on concise rapid reporting of clinical studies and reviews in COPD. Special focus is given to the pathophysiological processes underlying the disease, intervention programs, patient focused education, and self management protocols. This journal is indexed on PubMed Central, MedLine and CAS. The manuscript management system is completely online and includes a very quick and fair peer-review system, which is all easy to use. Visit http://www.dovepress.com/testimonials.php to read real quotes from published authors. 